\title{
LLIN Ownership, Utilization, and Malaria Prevalence: An Outlook at the 2015 Nigeria Malaria Indicator Survey
}

\author{
Jalal-Eddeen Abubakar Saleh*, Abdullahi Saddiq, Akubue Augustine Uchenna \\ Communicable Diseases Cluster, World Health Organization, Abuja, Nigeria \\ Email: *salehj@who.int
}

How to cite this paper: Saleh, J.-E.A., Saddiq, A. and Uchenna, A.A. (2018) LLIN Ownership, Utilization, and Malaria Prevalence: An Outlook at the 2015 Nigeria Malaria Indicator Survey. Open Access Library Journal, 5: e4280.

https://doi.org/10.4236/oalib.1104280

Received: December 21, 2017

Accepted: January 22, 2018

Published: January 25, 2018

Copyright $\odot 2018$ by authors and Open Access Library Inc.

This work is licensed under the Creative Commons Attribution International License (CC BY 4.0).

http://creativecommons.org/licenses/by/4.0/

\begin{abstract}
Background: Malaria is a disease caused by an infected female Anopheles mosquito. Nigeria, one of the two epicenters of malaria transmission across the globe, contributes to 40 percent global malaria burden. The disease, considered a global priority, has an annual global death toll of around 400,000 people. The global reduction in malaria burden which is the result of direct scale-up of one of the core malaria interventions using the insecticide-treated nets (ITNs), has prevented around 663 million cases of malaria in the subSaharan Africa. The most cost-effective evidenced-based strategy against malaria and its unwanted consequences is sleeping under the LLINs; studies have proven this measure to confer adequate protection to the mother and her unborn child. Methods: Quantitative cross-sectional study using secondary data obtained during the 2015 Nigeria malaria indicator survey. The eligible participants in the survey were pregnant women and caregivers who had at least a child under the age of five years. The survey was conducted in October and November 2015 across the thirty-six states of the country including the Federal Capital Territory. Using SPSS version 24, correlation and regression analyses were run to check for any significant correlation between LLIN use and malaria prevalence. Results: The Pearson correlation is 0.866 ( $\alpha=0.026$, $\mathrm{p}<0.05) 2$-tailed test, showing positive linear relationships between LLIN use and malaria prevalence across the six geo-political zones in 2015. The ANOVA test shows F value $21.510(\alpha=0.017, \mathrm{p}<0.05)$ and Regression analysis, R-square 0.935; these further shows a significant correlation between LLIN use and malaria prevalence. In favour of the Alternative Hypothesis, the Null Hypothesis was rejected. However, LLIN ownership and malaria prevalence shows weak correlation with a Pearson correlation of $0.635,(\alpha=0.175, \mathrm{p}<$ $0.05)$ 2-tailed test. Conclusion: The outcome of this study once again underscores LLIN as an important prevention tool against malaria and its unwanted
\end{abstract}


consequences. The positive linear correlation on LLIN use and the decline in malaria prevalence underscores the need for governments at all levels to ease up LLIN access to reduce any intervention gaps to further reduce malaria morbidity and mortality and achieve malaria elimination across the country.

\section{Subject Areas}

Public Health

\section{Keywords}

Malaria, Long Lasting Insecticidal Nets, Insecticide Treated Nets, Rapid

Diagnostic Test, Roll Back Malaria, Prevalence, Malaria Indicator Survey, Nigeria

\section{Background}

Malaria, a disease caused by an infected female Anopheles mosquito, is completely preventable, treatable and curable. The disease is considered not just a regional but global priority with a death toll of about 400,000 people globally every year [1] [2]. The most vulnerable group to malaria and its negative consequences are the pregnant women and children under the age of five years [3]. In pregnancy, malaria predisposes expectant mothers to an increased risk of anaemia, spontaneous abortions, stillbirths, premature deliveries, intra-uterine growth retardation and low birth weight babies, and these are all important causes of infant mortality. In the under-fives, anaemia is the commonest complications often resulting in a fatal outcome [4] [5] [6].

Although there are various types of malaria preventive measures, adherence to more than one measure often gives a better protection [1] [2]. In addition to malaria vaccine which will come into effect in some selected African countries in 2018, the other WHO recommended malaria preventive measures include the use of long-lasting insecticidal nets (LLIN), intermittent prophylactic treatment in pregnancy (IPTp), intermittent prophylactic treatment in infants (IPTi), indoor residual spray (IRS), and seasonal malaria chemoprophylaxis (SMC) [1] [2]. However, and especially in malaria endemic areas, the most cost-effective evidenced-based strategy against malaria and its unwanted consequences is sleeping under the LLINs; studies have proven this measure to confer adequate protection to the mother and her unborn child [4] [5] [7].

The LLINs are designed to be effective against mosquitoes for at least three years thus preventing malaria and all other mosquito-related illnesses. While some are skeptical about the use of the LLINs, several studies have proven their safety and effectiveness against malaria [2] [4]. The international recommendation by the WHO and Roll Back Malaria (RBM) partnership is universal coverage using free or heavily subsidized distribution of the LLINs through a feasible system that includes targeted mass campaigns [8] [9]. 
At least 80 percent of the global malaria burden is in Africa with the sub-Saharan region worst affected. While Nigeria and the Democratic Republic of Congo (DRC) have up to 40 percent of this figure, Nigeria alone contributes 29 percent of the over 80 percent global malaria burden [8]. In Nigeria, malaria contributes up to 60 percent of all outpatient visits, 30 percent of all hospital admissions, 11 percent of maternal mortality, 30 percent of under-five mortality and 25 percent of infant mortality [1] [4] [5]. In the sub-Saharan African, there are 43 percent of people who are at risk of malaria from mosquito bites and this is due to lack of access to the basic recommended core prevention tools-the long-lasting insecticidal nets and indoor spraying of insecticides [1] [4] [5].

Nigeria, with a population of 177.1 million [10], is the most populous country in Africa and occupying seventh position in the world. In Nigeria, there are about 110 million clinically diagnosed malaria cases and 300,000 malaria-related childhood deaths annually. Malaria in Nigeria, which already overburdens the already weakened health system, adversely affects the social and economic sectors of the country [10] [11]. With an annual decline in the country's GDP by 40 percent and nearly 480 billion naira annual out-of-pocket spending for treatments, prevention costs and loss of man hours, malaria negatively impacts on the economy [10] [11].

In the last decade, the global fight against malaria has recorded an appreciable success with the decline in the global malaria burden as mortality rate has dropped by 62 percent between 2000 and 2015 and 29 percent between 2010 and 2015 [1]. Similarly, the reduction in mortality due to malaria in children under the age of five is even more with about 69 percent drop between 2000 and 2015 and a further 35 percent between 2010 and 2015 [1]. In Nigeria, the story is not different as the record shows a decline in the national malaria prevalence from 42 percent in 2010 to 27 percent in 2015 [10]. Thus, the global reduction in malaria burden which is the result of direct scale-up of the core malaria interventions using the insecticide-treated nets (ITNs), has prevented around 663 million cases of malaria in the sub-Saharan Africa [1] [4] [5]. It is clear that for countries to achieve malaria elimination, there is need to make available not only the diagnostic and therapeutic components of the strategy but also to improve and ensure universal access to the critical malaria prevention tools; the long-lasting insecticide-treated nets (LLINs), preventive medicines in pregnant mothers (IPTp) and children under the age of one year (IPTi), seasonal malarial chemoprophylaxis (SMC) in the under-fives, and indoor residual spraying (IRS) [2].

There are studies that show strong correlation between mass LLIN use and a reduction in the prevalence of parasitaemia in pregnant women and children under the age of five years [12] [13] [14] [15]. However, in 2015, although the Nigeria malaria indicator survey have shown increase in LLIN/ITN ownership and use in all the six geo-political zones, this increase did not translate into a significant decline in malaria prevalence in some of the zones [10], hence the basis for this study to look if there is any significant correlation between LLIN/ITN use and malaria prevalence. 


\section{Method}

This quantitative cross-sectional study was retrospective in nature and used secondary data obtained from respondents during the 2015 Nigeria malaria indicator survey (MIS). The selection criteria for eligible participants in the survey were pregnant women and caregivers who have at least a child under the age of five years. The demographic and socio-economic profile of the respondents reveals 39 percent live in urban areas and 61 percent live in rural areas; 39 percent have no education, 16 percent have a primary school education, 36 percent have a secondary school education, and 10 percent have more than a secondary school education. Similarly, the proportion of women in the lowest wealth quintile is 18 percent and the highest quintile 23 percent [10]. The survey was conducted in October and November 2015 across the thirty-six states of the country including the Federal Capital Territory [10].

The research question and hypotheses for the study were:

RQ: What is the correlation between LLIN use and malaria prevalence?

Ho: There is no significant correlation between LLIN use and malaria prevalence.

Ha: There is a significant correlation between LLIN use and malaria prevalence.

The independent variable was LLIN use, and the dependent variable was malaria prevalence.

Using SPSS version 24, the study conducted correlation and regression analyses, using two-tail hypothesis testing and p-value 0.05 at $95 \% \mathrm{CI}$ to look if there was a significant correlation between LLIN use and malaria prevalence.

During the survey, participants were asked whether they slept the previous night under LLIN and then tested for malaria parasite using malaria rapid diagnostic test kit (RDT) and microscopy [10]. Approval on the use of the survey data was obtained from the DHS program administrator and downloaded from the website using the link provided. The DHS definitions used during the survey were- "LLIN ownership" meant reported household ownership of LLIN, "coverage" meant the LLIN owned were used accordingly, "LLIN use" meant the proportion of pregnant women who slept under an LLIN the previous night of the survey, and "universal coverage" implied that all members of a household slept under LLIN the previous night at two members per one LLIN [9] [10] [16].

\section{Results}

As shown in Figure 1, there was a significant improvement in LLIN ownership in Nigeria both at national and zonal levels in 2015. The increase in the national average of LLIN ownership from 42 percent in 2010 to 69 percent in 2015 had contributed to a drop in the national malaria prevalence from 42 percent in 2010 to 27 percent in 2015. Figure 2 and Figure 3 as well as Table 1 shows zonal ownership and use of the LLINs during the 2015 malaria indicator survey; the northwest zone had the highest ownership (90.6\%) and use (54.4\%) whereas the 


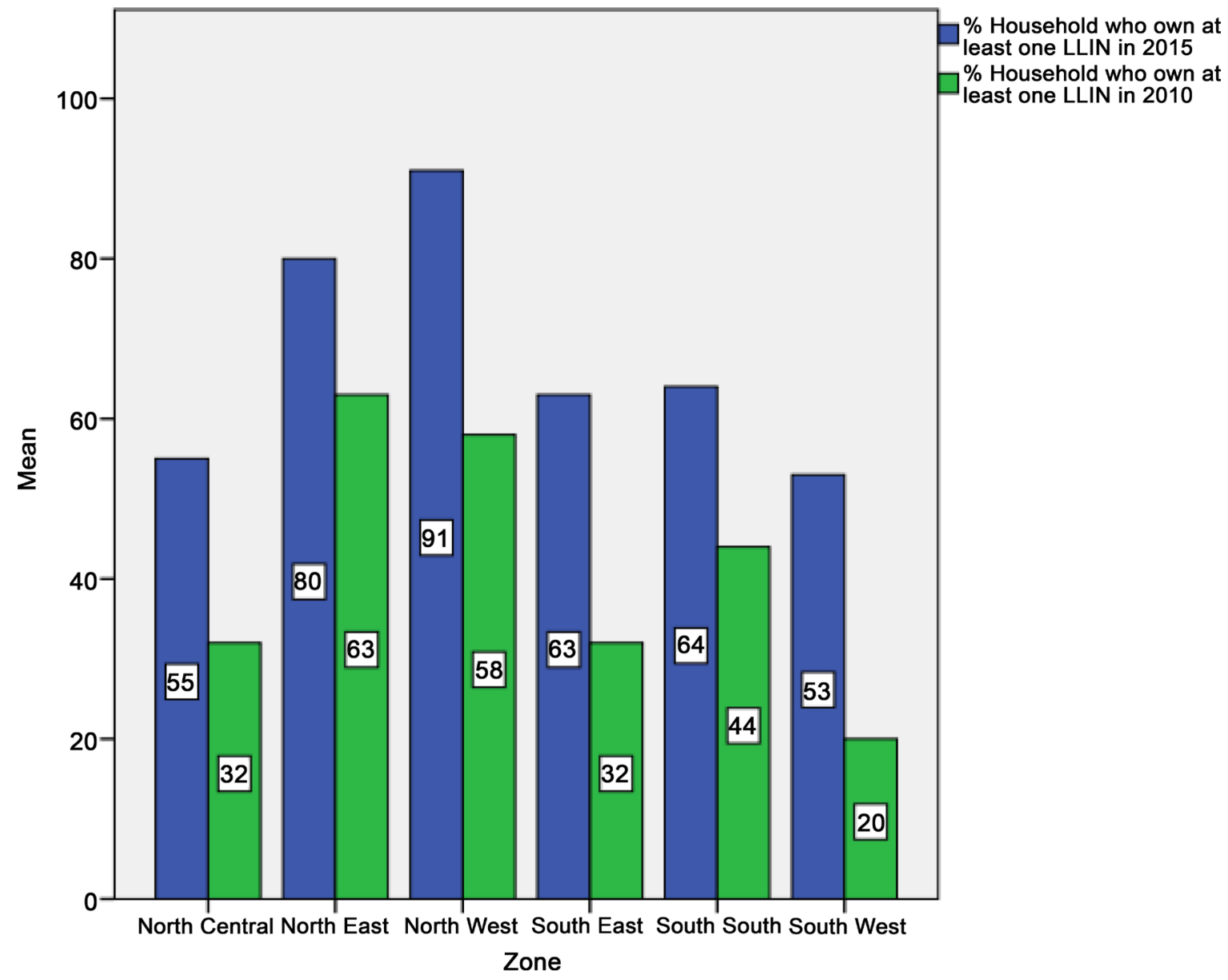

Figure 1. Bar chart showing LLIN ownership across the six geo-political zones in 2010 and 2015.

House ownership of at least one LLIN in 2015

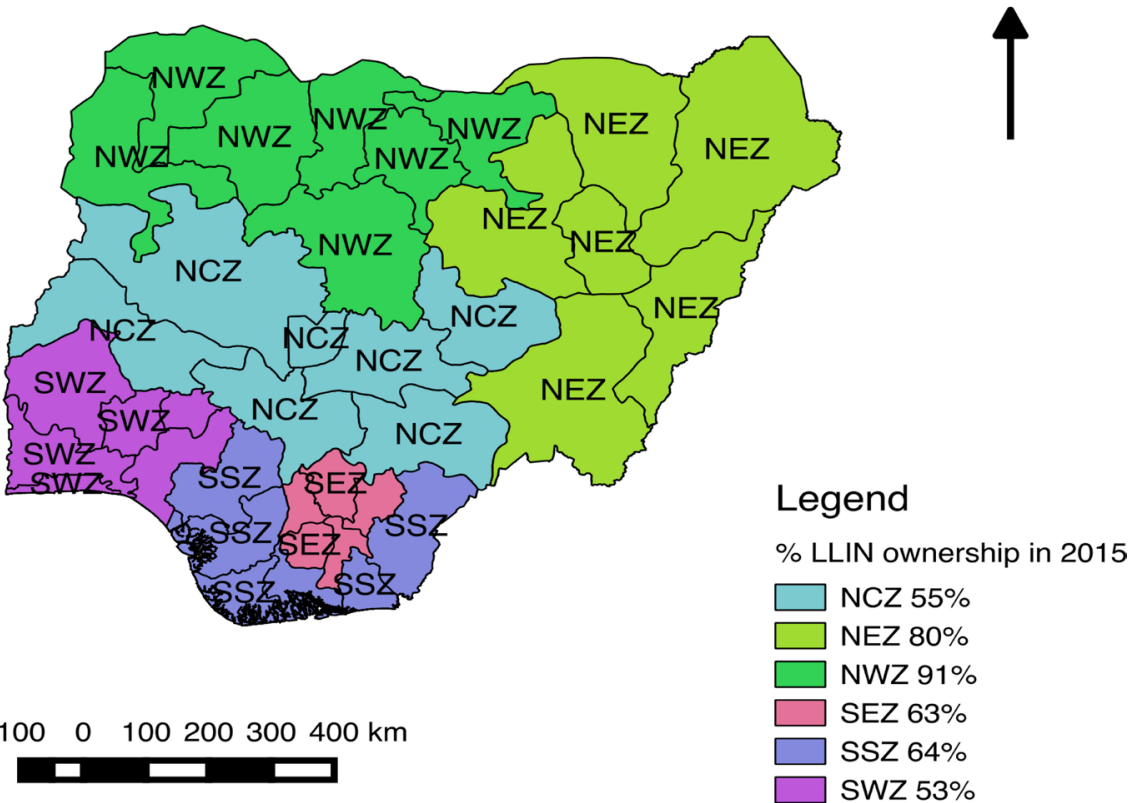

Figure 2. QGIS showing zonal ownership LLIN in 2015. 
$\%$ Population who slept inside an LLIN the night before survey in 2015

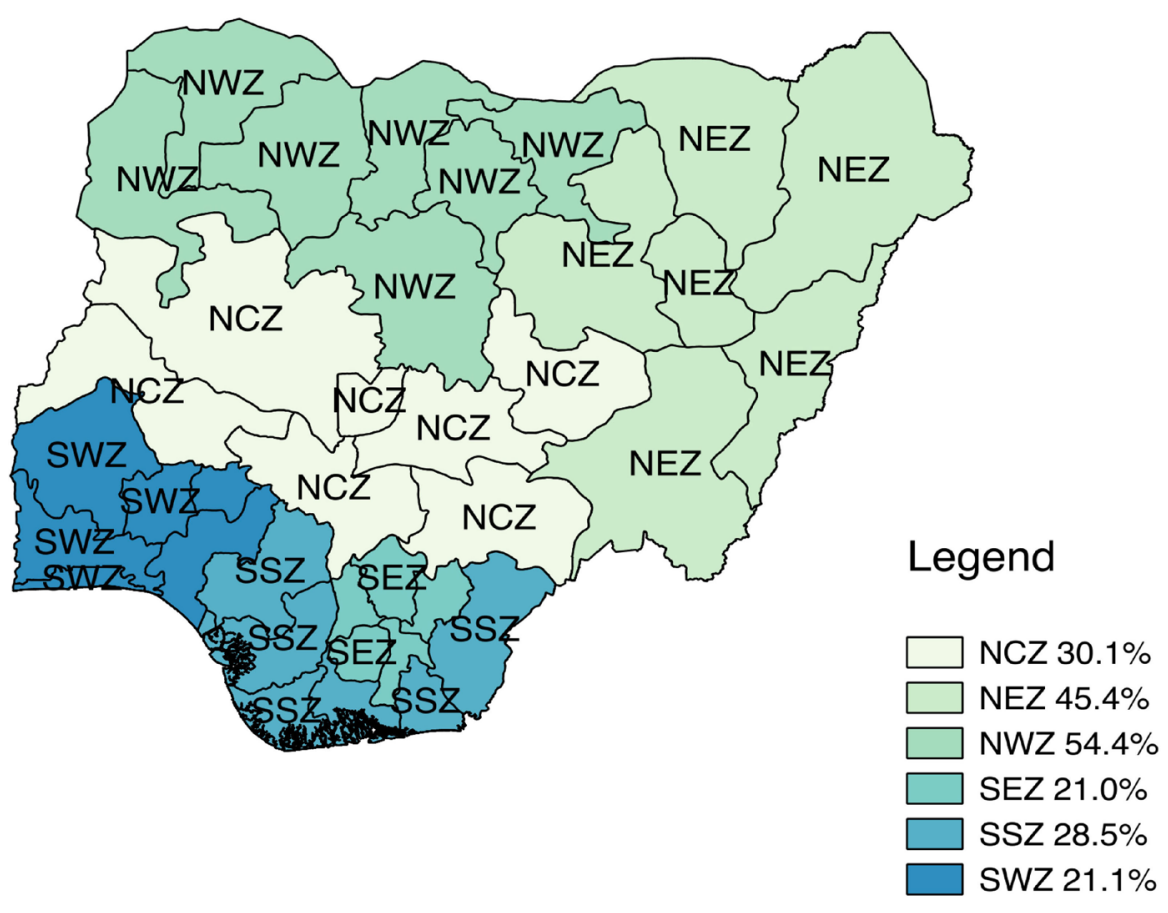

$100 \quad 0 \quad 100200300400 \mathrm{~km}$

Figure 3. QGIS showing zonal use of LLIN in 2015

Table 1. Descriptive statistics of LLIN ownership and use in 2015 across Nigeria.

\begin{tabular}{ccccccc}
\hline & $\begin{array}{c}\text { North } \\
\text { Central }\end{array}$ & $\begin{array}{c}\text { North } \\
\text { East }\end{array}$ & $\begin{array}{c}\text { North } \\
\text { West }\end{array}$ & $\begin{array}{c}\text { South } \\
\text { East }\end{array}$ & $\begin{array}{c}\text { South } \\
\text { South }\end{array}$ & $\begin{array}{c}\text { South } \\
\text { West }\end{array}$ \\
\hline $\begin{array}{c}\text { \% Households with at least one LLIN } \\
\text { in 2015 }\end{array}$ & 55.2 & 79.6 & 90.6 & 63.3 & 63.5 & 53.0 \\
$\begin{array}{c}\text { \% Population who slept inside an } \\
\text { LLIN the night before survey in 2015 }\end{array}$ & 30.1 & 45.4 & 54.4 & 21.0 & 28.5 & 21.1 \\
\hline
\end{tabular}

southwest zone and southeast had the least ownership (53.0\%) and use (21.0\%) respectively.

Although there was a reduction in malaria prevalence in the northwest zone from 48.2 percent in 2010 to 37.1 percent in 2015 due to improvement in the LLIN ownership and use as well as availability of other intervention measures, the northwest zone still had the highest malaria burden in the country (Figure 4). The increased commitment in malaria prevention in the southeast and southwest zones, doubling their LLIN ownership from 32.0percent and 20.0 percent in 2010 to 63.3 percent and 53.0percent in 2015 respectively (Figure 1), had significant impact on malaria infection with a reduction in prevalence from 27.6 percent and 50.3 percent in 2010 to 13.7 percent and 16.6 percent in 2015 
respectively (Figure 4).

In this study, the Null Hypothesis states that there is no correlation between LLIN use and malaria infection while the Alternative Hypothesis states that there is a significant correlation between LLIN use and malaria prevalence. The dots from the scatterplot in Figure 5 shows positive linear relationship between LLIN use and malaria prevalence across the six geo-political zones in 2015. This significant correlation is further supported by the Pearson Correlation $0.866, \mathrm{p}<$ 0.026, ( $\alpha$ 0.05, 2-tailed test) (Table 2). Thus, the Null Hypothesis was rejected and $t$ the Alternative Hypothesis which states that there is a significant correlation between LLIN use and malaria prevalence accepted. Conversely, the correlation is weak when we look at LLIN ownership and malaria prevalence as shown by the Pearson Correlation 0.635, $\mathrm{p}<0.175$ ( $\alpha$ 0.05, 2-tailed test) (Table 2).

Still, to support the Alternative Hypothesis is the Regression model which shows the R-square 0.935 and Durbin-Watson 1.988 (Table 3). Table 4 shows the ANOVA test with $\mathrm{F}$ value 21.510 at $\mathrm{df} 2$ and $\alpha 0.017$; this further supports a significant relationship between LLIN use and malaria prevalence thus rejecting the Null Hypothesis and accepting the Alternative Hypothesis.

Table 4 shows the ANOVA test with F value 21.510 atdf 2 and $\alpha 0.017$; this

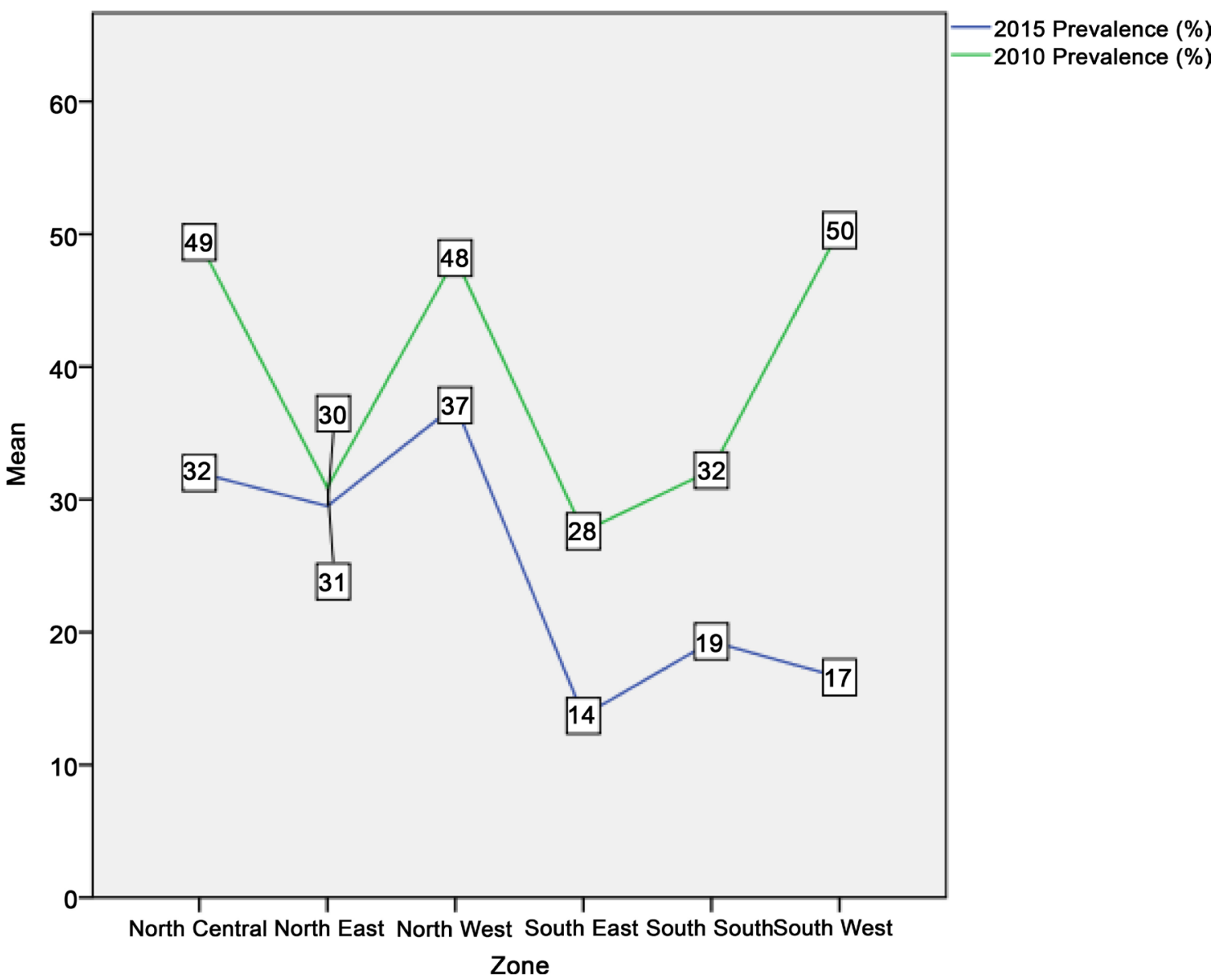

Figure 4. Line graph showing prevalence of malaria across the six geo-political zones in 2010 and 2015. 


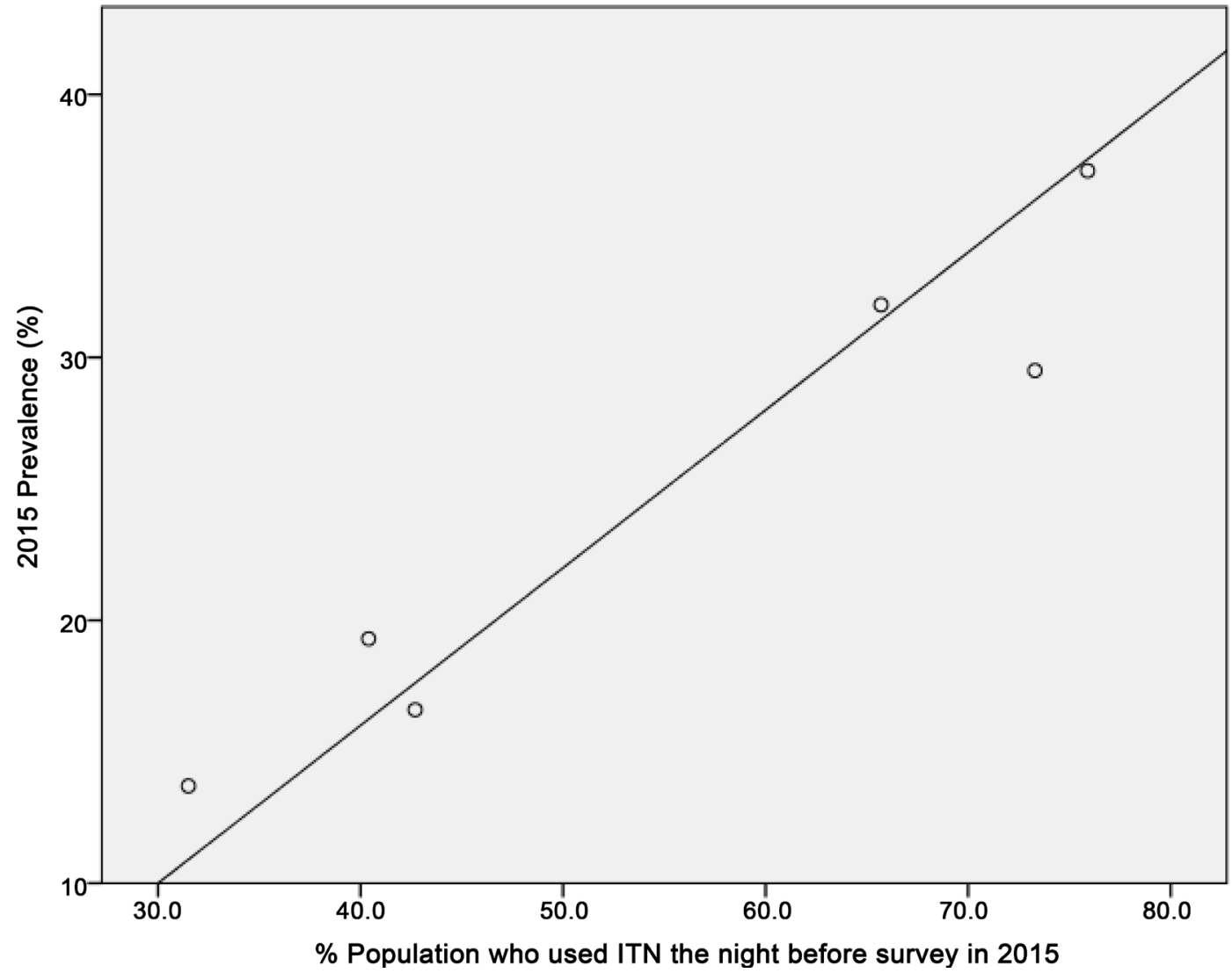

Figure 5. Scatterplot looking at relationship between malaria prevalence and LLIN use across the six geo-political zones in 2015.

Table 2. Correlation using 2-tailed of household who own at least one LLIN in 2015 and malaria prevalence in 2015 across Nigeria.

\begin{tabular}{|c|c|c|c|c|}
\hline \multicolumn{5}{|c|}{ Correlations $^{c}$} \\
\hline & & $\begin{array}{l}\% \text { of household who own } \\
\text { at least one LLIN in } 2015\end{array}$ & $\begin{array}{l}\text { \% Population who slept } \\
\text { inside an LLIN the night } \\
\text { before survey in } 2015\end{array}$ & $\begin{array}{l}2015 \text { Prevalence (slide } \\
\text { positivity rate) \% }\end{array}$ \\
\hline$\%$ of household who own at least & Pearson Correlation & 1 & $0.923^{* *}$ & 0.635 \\
\hline one LLIN in 2015 & Sig. (2-tailed) & & 0.009 & 0.175 \\
\hline$\%$ Population who slept inside an & Pearson Correlation & $0.923^{* *}$ & 1 & $0.866^{*}$ \\
\hline $\begin{array}{l}\text { LLIN the night before survey in } \\
\qquad 2015\end{array}$ & Sig. (2-tailed) & 0.009 & & 0.026 \\
\hline 2015 Prevalence (slide positivity & Pearson Correlation & 0.635 & $0.866^{*}$ & 1 \\
\hline rate) $\%$ & Sig. (2-tailed) & 0.175 & 0.026 & \\
\hline
\end{tabular}

${ }^{*}$ Correlation is significant at the 0.01 level (2-tailed). ${ }^{*}$. Correlation is significant at the 0.05 level (2-tailed). ${ }^{~}$ Listwise $\mathrm{N}=6$.

Table 3. Regression Model Summary of household who slept inside an LLIN the night before survey in 2015 and malaria prevalence (slide positivity rate) in 2015 across Nigeria.

\begin{tabular}{cccccc}
\hline \multicolumn{5}{c}{ Model Summary $^{\mathrm{b}}$} \\
\hline Model & $\mathrm{R}$ & R Square & Adjusted R Square & Std. Error of the Estimate & Durbin-Watson \\
\hline 1 & $0.967^{\mathrm{a}}$ & 0.935 & 0.891 & 3.1126 & 1.988
\end{tabular}

a Predictors: (Constant), \% Population who slept inside an LLIN the night before survey in 2015, \% Household who own at least one LLIN in 2015. ${ }^{b} \mathrm{De}-$ pendent Variable: 2015 Prevalence (slide positivity rate) \%. 
Table 4. ANOVA of household who slept inside an LLIN the night before survey in 2015 and malaria prevalence (slide positivity rate) in 2015 across Nigeria.

\begin{tabular}{ccccccc}
\hline \multicolumn{7}{c}{ ANOVA $^{\mathrm{a}}$} \\
\hline \multicolumn{1}{c}{ Model } & Sum of Squares & $\mathrm{df}$ & Mean Square & F & Sig. \\
\hline & Regression & 416.795 & 2 & 208.398 & 21.510 & $0.017^{\mathrm{b}}$ \\
1 & Residual & 29.065 & 3 & 9.688 & & \\
& Total & 445.860 & 5 & & & \\
\hline
\end{tabular}

a Dependent Variable: 2015 Prevalence (slide positivity rate) \%. 'Predictors: (Constant), \% Population who slept inside an LLIN the night before survey in 2015, \% Household who own at least one LLIN in 2015.

further supports a significant relationship between LLIN use and malaria prevalence thus rejecting the Null Hypothesis and accepting the Alternative Hypothesis.

\section{Discussions}

The use of LLINs especially in malaria endemic countries is considered as one of the most cost-effective measures towards malaria prevention. This study, which critically examined the correlation between LLINs use and malaria prevalence in Nigeria in 2015, has shown a strong correlation. Although there were appreciable improvements across the country regarding the decrease in malaria prevalence vis-à-vis LLIN use in 2015, the northwest zone which had the highest ownership did not register a significant decline in malaria prevalence when the 2010 and 2015 malaria indicator surveys were compared.

However, while several studies have underscored the use of LLIN as an important preventive tool against malaria infection, there are studies that show deficits between the LLIN ownership and use [17] [18]. While LLIN ownership was dependent on factors such as marital status, education, and knowledge of malaria or ITNs/LLINs, factors that promote LLIN use were not centered on only adequate education and knowledge on malaria and ITNs/LLINs, but also on household income, socio-economic status and whether residing in urban areas [19]. Equally, in studies conducted in Nigeria and Uganda, results showed a low level of education as a factor to higher rates of ITN use [20] [21]. The reason to this could be that the lower educated ones perceived a greater threat to malaria than the most educated ones. Often ownership did not translate to use of the LLINs for reasons that include uneasiness, heat, a misconception on the benefit of ITNs, and preference for other preventive tools [20] [21].

In the 2015 Nigeria malaria indicator survey, despite the high LLIN ownership in the northwest zone, theplausible explanations to the low LLIN use could be due to poor knowledge on LLIN/ITN use, lack of perceived threat to malaria by individuals or both. This was also the case in some countries where in spite the high ownership of LLIN/ITNs, the use was poor [22].

\section{Conclusion}

The outcome of this study once again underscores LLIN as an important pre- 
vention tool against malaria and its unwanted consequences. The positive linear correlation on LLIN use and the decline in malaria prevalence from the 2015 Nigeria malaria indicator survey from across the six geopolitical zones calls on the need for governments at all levels to ease and increase access to the LLINs by individuals and especially to the most vulnerable groups to reduce malaria morbidity and mortality and improve the country's health indicators. Similarly, there is need for increased emphasis on behavioural change communication strategies and to educate the populace on ideal way to use the LLINs/ITNs to help increase the utilisation. Post distribution educational campaign should be incorporated into future distribution campaigns to help increase net utilisation.

\section{Acknowledgements}

We acknowledge with gratitude the approval for the use of data from DHS program administrator.

We declare that there is no any potential conflict of interest or royalty associated with the manuscript.

We declare that no funding is received from any source for this outbreak investigations.

\section{References}

[1] World Health Organization (2016) WHO Global Malaria Programme, World Malaria Report. WHO Document Production Service, Geneva. http://www.who.int/malaria/publications/world-malaria-report-2016/en/

[2] World Health Organization (2017) World Malaria Day. http://www.who.int/campaigns/malaria-day/2017/en/

[3] World Health Organization (2011) World Malaria Report 2011. World Health Organization, Geneva.

[4] WHO Global Malaria Programme (2016). http://www.who.int/malaria/en/

[5] GTS (2016) Global Technical Strategy for Malaria 2016-2030. http://www.who.int/malaria/publications/atoz/9789241564991/en/

[6] WHO (2004) A Strategic Framework for Malaria Prevention and Control during Pregnancy in the African Region. World Health Organization: World Health Organization, Regional Office for Africa, Geneva.

[7] Kyu, H.H., Georgiades, K., Shannon, H.S. and Boyle, M.H. (2013) Evaluation of the Association between Long-Lasting Insecticidal Nets Mass Distribution Campaigns and Child Malaria in Nigeria. Malaria Journal, 12, 14. https://doi.org/10.1186/1475-2875-12-14

[8] World Health Organization (2014) WHO Global Malaria Programme, World Malaria Report. WHO Document Production Service, Geneva.

[9] RBM (2009) Guidelines for Core Population-Based Indicators. RBM Technical Paper Series No. 1. Roll Back Malaria, Geneva.

[10] NMEP, NPopC, NBS, and ICF International (2016) Nigeria Malaria Indicator Survey 2015. NMEP, NPopC, and ICF International, Abuja and Rockville.

[11] NMEP (2014) National Malaria Strategic Plan 2014-2020. NMEP and Roll Back Malaria, Abuja. 
[12] Gamble, C.L., Ekwaru, J.P. and terKuile, F.O. (2009) Insecticide-Treated Nets for Preventing Malaria in Pregnancy. The Cochrane Database of Systematic Reviews, No. 2, CD003755.

[13] Eisele, T.P., Larsen, D.A., Anglewicz, P.A., Keating, J., Yukich, J., Bennett, A., Hutchinson, P. and Steketee, R.W. (2012) Malaria Prevention in Pregnancy, Birthweight, and Neonatal Mortality: A Meta-Analysis of 32 National Cross-Sectional Datasets in Africa. The Lancet Infectious Diseases, 12, 942-949. https://doi.org/10.1016/S1473-3099(12)70222-0

[14] Killeen, G.F., Smith, T.A., Ferguson, H.M., Mshinda, H., Abdulla, S., Lengeler, C. and Kachur, S.P. (2007) Preventing Childhood Malaria in Africa by Protecting Adults from Mosquitoes with Insecticide-Treated Nets. PLOS Medicine, 4, e229. https://doi.org/10.1371/journal.pmed.0040229

[15] Curtis, C.F., Jana-Kara, B. and Maxwell, C.A. (2003) Insecticide Treated Nets: Impact on Vector Populations and Relevance of Initial Intensity of Transmission and Pyrethroid Resistance. Journal of Vector Borne Diseases, 40, 1-8.

[16] ICF International (2012) Demographic and Health Survey Interviewer's Manual. Measure DHS Basic Documentation No. 2, ICF International, Maryland.

[17] Woyessa, A., Deressa, D., Ali, A. and Lindtjørn, B. (2014) Ownership and Use of Long-Lasting Insecticidal Nets for Malaria Prevention in Butajira Area, SouthCentral Ethiopia: Complex Samples Data Analysis. BMC Public Health, 14, 99. http://www.biomedcentral.com/1471-2458/14/99 https://doi.org/10.1186/1471-2458-14-99

[18] Aderibigbe, S.A., Olatona, F.A., Sogunro, O., Akande, T.M., et al. (2014) Ownership and Utilisation of Long Lasting Insecticide Treated Nets following Free Distribution Campaign in South West Nigeria. Pan African Medical Journal, 17, 263. https://doi.org/10.11604/pamj.2014.17.263.3927

[19] Singh, M., Brown, G. and Rogerson, S.J. (2013) Ownership and Use of Insecticide-Treated Nets during Pregnancy in Sub-Saharan Africa: A Review. Malaria Journal, 12, 268. http://www.malariajournal.com/content/12/1/268 https://doi.org/10.1186/1475-2875-12-268

[20] Auta, A. (2012) Demographic Factors Associated with Insecticide Treated Net Use among Nigerian Women and Children. North American Journal of Medical Sciences, 4, 40-44. https://doi.org/10.4103/1947-2714.92903

[21] Ahmed, S.M. and Zerihun, A. (2010) Possession and Usage of Insecticidal Bed Nets among the People of Uganda: Is BRAC Uganda Health Programme Pursuing a Pro-Poor Path? PLoS ONE, 5, e12660. https://doi.org/10.1371/journal.pone.0012660

[22] Deribew, A., Alemseged, F., Birhanu, Z., Sena, L., Tegegn, A., Zeynudin, A., Dejene, T., Sudhakar, M., Abdo, N. and Tessema, F. (2010) Effect of Training on the Use of Long-Lasting Insecticide-Treated Bed Nets on the Burden of Malaria among Vulnerable Groups, South-West Ethiopia: Baseline Results of a Cluster Randomized Trial. Malaria Journal, 9, 121. https://doi.org/10.1186/1475-2875-9-121 